\title{
Hidroeléctrica Ituango, un caso para el análisis ambiental del territorio
}

\author{
Ituango Hydroelectric Plant, a Case for the Environmental Analysis of the Territory
}

Itzamar Nataly Cuervo López ${ }^{1}$

${ }^{1}$ Universidad Nacional de Colombia. Email: incuervol@unal.edu.co.

\section{OPEN ACCESS}

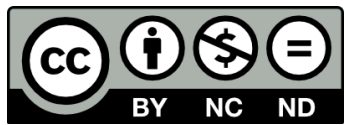

Copyright:(C) 2019 Ingenierías USBmed. La revista Ingenierías USBmed proporciona acceso abierto a todos sus contenidos bajo los términos de la licencia creative commons Atribución- no comercial- SinDerivar 4.0 Internacional (CC BY-NC-ND 4.0)

Tipo de Artículo: Investigación científica y tecnológica.

Recibido:31-05-2019.

Revisado: 29-07-2019.

Aprobado: 4-08-2019.

Doi: $10.21500 / 20275846.4139$

Referenciar así: I.N. Cuervo. "Hidroeléctrica Ituango, un caso para el análisis ambiental del territorio". Ingenierías USBMed, 10(2), pp.44-54, 2019 .

Declaración de disponibilidad de datos: Todos los datos relevantes están dentro del artículo, así como los archivos de soporte de información.

Conflicto de intereses: los autores han declarado que no existen conflicto de intereses.

Editores: Yohana López Rivera, Universidad de San Buenaventura, Medellín, Colombia. Alfonso Insuasti Rodríguez, Universidad de San Buenaventura, Medellín, Colombia. Erika Solange Imbett Vargas, Instituto Tecnológico Metropolitano. Eliana Zapata Ruiz, Instituto Tecnológico Metropolitano. José Fernando Valencia Grajales, Universidad Autónoma Latinoamericana.
Resumen. A partir de la investigación "Factores de desarrollo local en un hábitat afectado por proyectos de desarrollo hidroeléctrico: proyecto Hidroeléctrico Ituango, Antioquia, Colombia", desarrollada por la autora entre los años 2015 y 2018, este artículo plantea una lectura ambiental de los territorios afectados por proyectos hidroeléctricos. Desde una mirada a las normativas, el funcionamiento de los proyectos y la construcción social, se elabora un panorama de las condiciones de desarrollo promovidas por los megaproyectos, en el que se identifican algunas de las inconsistencias de tipo metodológico que se presentan durante la planeación y ejecución de los proyectos. A partir de este ejercicio se proponen ciertos ejes de análisis ambiental cuyo objetivo es ubicar el desarrollo local dentro de los marcos normativos, así como las metodologías empleadas por los proyectos hidroeléctricos una vez intervienen en los territorios. Estos ejes definen orientaciones para la comprensión de territorios afectados en la actualidad, y como ruta de atención a otras problemáticas que enfrentan las comunidades y administraciones locales en la implementación de grandes proyectos de desarrollo en Colombia y el mundo.

Palabras Clave. Territorio, desarrollo local, hidroeléctricas, megaproyectos, energía, comunidades, normativas

Abstract. Based on the research "Factors of Local Development in a Habitat Affected by Hydroelectric Development Projects: Ituango Hydroelectric Project, Antioquia, Colombia," developed by the author between 2015 and 2018. This article poses an environmental defense of the territories affected by hydroelectric projects. From a look at the regulations, the functioning of projects, and the social construction, an overview of the conditions of development promoted by megaprojects are prepared, which identifies some of the inconsistencies of methodological type, which are presented during the planning and execution of projects. From this exercise, certain lines of environmental analysis are proposed, which aim to locate the local development within the regulatory frameworks, as well as the methodologies employed by hydroelectric projects once involved in the territories. These lines define the guidelines for the understanding of territories affected at present, and as an assistance to other issues, which communities and local authorities in the implementation of major development projects face, in Colombia and the world.

Keywords. Territory, local development, hydropower, mega-projects, energy, communities, regulations. 
Nomenclatura

EIA: Estudios de Impactos Ambientales

ANLA: Autoridad Nacional de Licencias Ambientales

UPME: Unidad de planeación Minero Energética

PHI: Proyecto Hidroeléctrico Ituango

EPM: Empresas Públicas de Medellín

DNP: Departamento Nacional de Planeación

\section{Introducción}

El panorama actual del cambio climático, las pronunciadas desigualdades sociales y el riesgo que este representa para la naturaleza (su intensiva intervención), y con ella, para la humanidad, sitúa la construcción de megaproyectos de infraestructura y las consecuencias de afectación a los ecosistemas y a los tejidos sociales a nivel mundial, frente a la necesidad de conocer el alcance que tienen los proyectos de tipo hidroeléctrico respecto al desarrollo local de los territorios; de modo específico, cómo sostener y potenciar junto a las comunidades los factores de desarrollo construidos históricamente en el territorio.

Resulta importante la construcción de ejes de análisis que deriven del hábitat humano, entendido como una conjugación de la vida social, ambiental y política que haga posible el desarrollo de cada ser en el espacio según sus ritmos y su relación con la naturaleza [1], desde una articulación entre los ejes de análisis ambiental de las comunidades y los productores locales con sus administraciones (municipales y regionales) para megaproyectos como las centrales hidroeléctricas.

Es una prioridad renovar las actuales metodologías y lineamientos definidos para intervenir los territorios. Así, corresponde partir desde una perspectiva ambiental que reconozca el territorio, a través de la cual se comprendan la naturaleza y los procesos de construcción social de las comunidades como ejes centrales de desarrollo.

Los megaproyectos implican procesos previos a su resultado o finalidad - producción de energía, construcción de puertos o aeropuertos-. Desde la evaluación de sostenibilidad de proyectos de desarrollo existen etapas relacionadas con el análisis de desempeño y los impactos de los proyectos [2], las cuales son fundamentales para garantizar su avance. Al respecto, este artículo plantea una mirada al conjunto de procesos que implica un proyecto como Hidroituango en términos de ejes para el análisis ambiental del territorio.

\section{Los proyectos de desarrollo hidroeléctrico}

Los proyectos hidroeléctricos son actividades productivas que generan más interés para los inversionistas públicos y privados. Según el World Energy Council, este sector aporta al mercado el $16.4 \%$ de la energía mundial [3]. Dado que hace parte de las energías ren- ovables, se promueve como una de las fuentes viables desde lo ambiental para la producción de electricidad, a pesar de que su construcción interrumpe cerca del $60 \%$ de los ríos del mundo [4]. Lo anterior cuestiona la viabilidad económica de estos proyectos respecto a las afectaciones causadas al ambiente y a las comunidades, las cuales resultan de carácter irreversible para los ríos y los ecosistemas enteros.

Los estándares o criterios para el tratamiento de los afectados por proyectos hidroeléctricos derivan de lineamientos preestablecidos por sus promotores o ejecutores. En Colombia, la Metodología general para la Elaboración y Presentación de Estudios Ambientales precisa que estos deben atender los lineamientos de la metodología y términos de referencia, respondiendo de manera adecuada y responsable a los impactos ambientales [5].

Antes del Proyecto Hidroeléctrico Ituango, EPM había empleado las metodologías de política ambiental corporativa en el 2000, y de Responsabilidad social empresarial en el 2006 [6]. Respecto al proyecto hidroeléctrico Ituango, se decidió emplear las metodologías y lineamientos que definen los organismos multilateras y los bancos - como el BID-.

La revisión de estas metodologías y los términos de referencia para proyectos hidroeléctricos [7], a partir de los cuales se hace seguimiento a las intervenciones y afectaciones que los proyectos generan, han representado un avance respecto a las primeras hidroeléctricas de la década de 1960, por cuanto se reconocen variables ambientales y sociales. Sin embargo, no alcanzan una valoración conjunta del territorio pues este se separa en términos de biótico, abiótico y social, con lo que se condiciona el análisis de los lugares desde su diversidad ambiental y sociocultural, y se pierde su visión integral.

En el caso que nos ocupa, las comunidades debieron limitar sus peticiones a los requerimientos de la empresa ejecutora y a su proceso de negociación, en el cual se encontraba el Manual de Valores Unitarios [8] editado por EPM, cuyo tratamiento individualizado para la valoración de predios y actividades económicas debilitó aún más el tejido comunitario y la capacidad de integración de las comunidades en los territorios intervenidos.

Una de las problemáticas más claras en la implementación de proyectos hidroeléctricos radica en la aparición de las comunidades como un actor secundario en la planificación y alcance de los procesos de desarrollo de su territorio. En el caso de Hidroituango se dio un proceso de socialización con las comunidades que se limitó a la trasmisión de información sobre las condiciones técnicas y programas de intervención del proyecto; empero, esto no implicó una oportunidad para la inclusión de las aspiraciones de desarrollo local, dada la baja articulación del proyecto con las organizaciones 
comunitarias y los municipios, sumada a la subestimación de las capacidades del territorio en términos de producción local, reservas ambientales y contenido histórico y patrimonial. Se evidenció la falta de control sobre los impactos del proyecto hidroeléctrico en el área de influencia, que dio origen la solicitud de la ANLA, en febrero de 2019, de ejecutar medidas de protección para frenar el daño ambiental y social.

Las metodologías implementadas en Colombia por las autoridades ambientales y las definidas por la legislación ambiental (leyes 388 de 1997 y 152 de 1994 y decretos 1320 de 1998 sobre la consulta previa y 2041 de 2014 sobre licenciamientos ambientales), tienen en su contenido la instancia de participación de las comunidades. Según el Decreto 2041 de 2014, se debe informar sobre estas con énfasis en los impactos y medidas de manejo, a la vez que en dicha actividad se deben incorporar los aportes que resulten del proceso de participación cuando se consideren pertinentes.

No obstante, la decisión de organismos que actúan por fuera del alcance local, como los planes de desarrollo nacional o continental (IIRSA, Conectando las Américas 2022 e Interconexión Colombia-Panamá) [9] ha rezagado las ventajas comparativas de estos lugares, hecho que causa un abandono sistemático en función de la homogenización en los usos del suelo a la producción de energía y, con ello, la negación de infraestructura básica - conectividad vial, salud y educación- esencial para el desarrollo en el ámbito local.

Los proyectos hidroeléctricos parten de desigualdades existentes en el territorio, tales como el bajo desarrollo social, y la mínima respuesta organizativa de las comunidades. Por esto no se da una equivalencia entre los argumentos y discursos de los proyectos de desarrollo con las demandas de la comunidad para la protección ambiental.

Hidroituango concibe y predispone el territorio a un fin único -la producción de energía-, a la vez que articula los procesos económicos globales con las dinámicas locales. Esto afecta la naturaleza sin que signifique una conexión real con las dinámicas sociales locales, es decir, con sus capacidades productivas y culturales. Cuando se instala el proyecto predomina un tejido de decisiones en el que los campesinos, mineros y pescadores, ven transformada su realidad cotidiana en las veredas y los poblados; esperan mejorar sus estándares de subsistencia dado que el proyecto promete oportunidades de trabajo en servicios, turismo y comercio, las cuales pueden demorar años en materializarse o, incluso, no alcanzar las expectativas.

\section{A. Hidroituango como referente de análisis}

El ejercicio académico propuesto parte del acercamiento a la experiencia del Proyecto Hidroeléctrico Ituango, localizado en el departamento de Antioquia (Colombia), el cual se encuentra en fase de planeación a la fecha de elaboración del presente artículo. Su objetivo es producir $2400 \mathrm{MW}$ al año 2021, de tal forma que se atienda el $17 \%$ de la demanda actual de energía eléctrica del país [10].

El Proyecto Hidroeléctrico Ituango, considerado el más grande de su tipo en el país, lleva a cabo su construcción en el territorio donde se localiza bajo el argumento de generación de desarrollo para los municipios donde tendrían lugar sus intervenciones: se beneficiarían con ello 12 municipalidades con programas de atención en las líneas de infraestructura educativa, vial y construcción de vivienda. Sin embargo, desde abril del año 2018, la empresa EPM, constructora del proyecto, registró fallas técnicas que obligaron a declarar en emergencia el área de influencia del mismo, dadas las afectaciones ambientales y el riesgo que representa el avance de su construcción. Se definió entonces un estado de contingencia [11] en el que hasta la fecha se registran 23 municipios afectados, incluidos los departamentos de Córdoba y Sucre que se encuentran aguas abajo.

A partir de entonces, y al evidenciarse las dificultades técnicas y socioambientales del proyecto presenta, la hidroeléctrica ha permanecido bajo la mirada de la ciudadanía. Esto marcó una preocupación por el alcance de las afectaciones y por la promesa incumplida de desarrollo para las comunidades, que ha significado y hasta entonces se ha configurado en incertidumbres y dudas por el futuro del territorio.

Surgieron así preocupaciones por la estabilidad del proyecto de parte de distintos actores del territorio, cuyo objetivo era ejercer permanente veeduría al proyecto, una vez "el Tribunal Superior de Medellín ordenó al gobierno nacional ejercer la tutoría y representación legal [énfasis propio] sobre los derechos del Río Cauca" [12]. El Proyecto Hidroeléctrico Ituango revela la actual crisis ambiental, pues ha exigido un análisis de las condiciones socioambientales que intervienen en los territorios; se logró con ello identificar tres problemáticas desde la ingeniería, la política y la economía, pues de estas depende la materialización del desarrollo en un territorio.

El Río Cauca es un ejemplo la manera como la ingeniería es capaz de transformar un territorio en función de la creciente demanda sobre el recurso hídrico para la generación de energía. No obstante, la ingeniería no se reduce a la técnica, pues las decisiones políticas y los ritmos de la economía global han sometido a las comunidades a los acelerados cambios de la producción a gran escala.

Se han modificado y sacrificado importantes ecosistemas y comunidades para cubrir la demanda de energía de las ciudades y la industria nacional e internacional, sin plantear oportunidades claras para el territorio que a largo plazo garanticen la protección de la fauna, la flora y la continuidad de actividades 
productivas sostenibles. Es decir, no existe certeza de que van a permanecer los mínimos sistemas biológicos y humanos para proveer a las generaciones futuras.

El proceso de desarrollo de Hidroituango ha configurado un escenario de incertidumbres que ha comprometido tanto el carácter técnico-civil como la responsabilidad social del proyecto hidroeléctrico. Así, la declaratoria del 20 de junio de 2019 del Río Cauca como sujeto de derechos busca, además de la protección de la cuenca, la salvaguarda de un ambiente sano y seguridad alimentaria para las generaciones futuras que habiten este territorio.

Se plantea, con todo lo dicho, una necesidad de incorporar la ingeniería en los procesos de desarrollo, a fin de entender integralidad de la técnica, la economía y la política, y visualizarlos más allá de los estándares y criterios preestablecidos para la ejecución de un proyecto.

B. Proceso de desarrollo de los proyectos hidroeléctricos La mayoría de los proyectos hidroeléctricos construidos en el mundo registran marcas negativas en los entornos donde intervienen. Esta premisa permite ir más allá de la descripción de impactos, ya abordados con suficiencia por otros investigadores e instituciones en distintos escenarios.

Autores como Patrick McCully [13] y Chellaney [14] plantean la disputa por el recurso hídrico y las afectaciones a la naturaleza y a los entornos socioculturales como una de las consecuencias directas de la búsqueda incansable por el desarrollo económico y el costo del "progreso". En Colombia, por ejemplo, se suman estudios de proyectos hidroeléctricos sobre la Hidroeléctrica el Quimbo [15] e Hidrosogamoso [16]: estos casos confirman, al igual que Hidroituango, el declive del territorio y la incertidumbre que generan entre las comunidades y hacia el ecosistema. A su turno, y en relación con el caso del proyecto hidroeléctrico previsto sobre el Río Samaná Norte, Hoyos y Rodríguez muestran la posible pérdida de especies endémicas y desconocidas, inclusive por los estudios hasta ahora recibidos por la Autoridad Nacional de Licencias Ambientales [17].

La integración de las comunidades al desarrollo que ofrecen los proyectos hidroeléctricos y sus infraestructuras obedece a la relación entre funcionalidad de los proyectos y necesidades inmediatas de los sectores económicos: construcción, energía e infraestructura.

Los proyectos hidroeléctricos en Colombia se plantean con 20 años o más de anterioridad, pues la identificación de potenciales hidroeléctricos es una tarea constante de los ministerios a cargo del sector energético (hoy amparado por la Ley 1715 de 2014, la cual busca el aprovechamiento de energía no renovable y la exploración de dichas fuentes). Se identificó el potencial en el río Cauca, a la altura de Ituango, en el año 1967; desde entonces se consideró un entorno para la producción de energía, y su materialización inició en 2009.
Durante este proceso el uso del suelo quedó sometido a disposición exclusiva del proyecto: con él surgieron expectativas en función de la producción minero-energética, de la cual dependería el futuro del territorio.

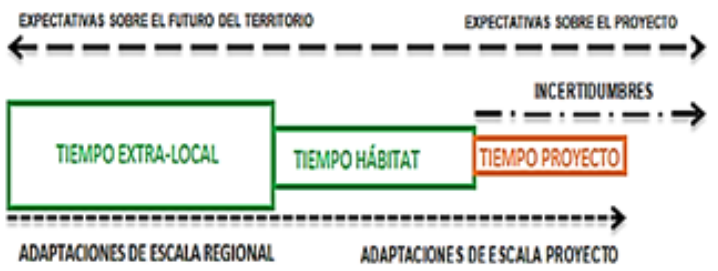

Figura 1. Combinación de temporalidades y ritmos en el hábitat afectado [6]

En la figura 1 se esquematizan las temporalidades en relación con la escala de intervención del proyecto. El llamado tiempo extra local -es decir, el tiempo de los planes y del gobierno- abarca la mayor cantidad de tiempo y espacio. Lo sigue el tiempo del hábitat, en el que aparecen el territorio, sus comunidades y ecosistemas: tiene que ver con las actividades locales, y suele estar sometido a presiones de agentes externos y al impulso del proyecto hidroeléctrico. A este lo sigue la ejecución del proyecto: si bien esta incide en menor medida durante la construcción, en adelante es artífice de transformación del territorio en tanto genera incertidumbres y lo somete a múltiples modificaciones y necesidades constructivas: vías, servicios, provisión de mano de obra, oferta de comercio y acogida a la población flotante que se ve atraída por el proyecto.

Estos cambios suponen una aceleración de la economía en el territorio; empero, una vez entran en tensión los actores de inversión y ejecución con las comunidades relacionadas, emergen procesos de resistencia y organización social cuyo interés principal es la negociación con las empresas ejecutoras y la reclamación de derechos adquiridos. Además, estos buscan la inclusión en la toma de decisiones sobre aquello que les afecta para incidir en los planes o programas a implementarse, en aras de un proyecto que tiene una vida útil de 50 a 150 años. Por lo tanto, los efectos sobre los ecosistemas y la sostenibilidad local son determinantes en la continuidad del territorio.

En línea con lo anterior, movimientos como Ríos Vivos y Asopesca, y diversas asociaciones de mineros, barequeros y pescadores de la región, han movilizado esfuerzos para defender sus actividades económicas y proteger el entorno que habitan. Esto ha traído consigo nuevas solicitudes de las comunidades en cuanto a los problemas ambientales que afectan el territorio, de manera que la estabilidad de la presa y las garantías técnico-constructivas del proyecto adquieren igual importancia que las garantías de trabajo, vivienda y bienestar de los habitantes del área de influencia directa e indirecta del proyecto. 
La hegemonía de los sectores productivos sobre los territorios se manifiesta en alteraciones de las relaciones cotidianas y, más adelante, en su ruptura o reemplazo. Ello da lugar a la circulación del capital y la conversión de los territorios en mercancías adaptadas al mercado global.

C. Propósitos de desarrollo de las hidroeléctricas en el territorio

Con la apertura económica ocurrida hacia finales de la década de 1980, el desarrollo de infraestructura se consideró el camino efectivo para contrarrestar los niveles crecientes de pobreza, mediante un discurso que favorecía el desarrollo de las comunidades aisladas de los principales centros urbanos de países de América Latina. Sin embargo en el mismo periodo el uso y disposición de la base natural y las instituciones del Estado se ancló en el sector privado, lo cual situó los servicios públicos en asocio con los particulares; desde entonces, la posibilidad de cerrar la brecha de desigualdad a través de megaproyectos se ha hecho más contundente.

Las primeras centrales hidroeléctricas que se construyeron en Colombia se realizaron con fines exclusivos de interconexión eléctrica en el año 1946, en respuesta a una demanda de infraestructura para la provisión de energía y agua a los centros urbanos. Allí, los ámbitos sociales y ambientales de los proyectos quedaron relegados a la idea de desarrollo de entonces: una intervención funcional a través de la cual la infraestructura resolvería parte de las problemáticas existentes.

Solo hasta 1973 se generaron estudios de impacto ambiental en el país mediante la implementación del código de Recursos Naturales pionero en Latinoamérica, dispuesto en el artículo 69 del Decreto 2811 de 1974:

(...) Uso eficiente de recursos hídricos y obras hidráulicas de propiedad privada $(\cdots)$ establecimiento, mejora, rehabilitación y conservación de servicios públicos concernientes al uso de aguas, tales como suministro de estas, alcantarillado y generación de energía eléctrica [18].

Es decir, un enfoque de uso y disposición de la base hídrica como recurso de consumo y generación de servicios. Así, la construcción de centrales hidroeléctricas, constituye una forma de adquisición de bienes para la defensa de recursos naturales, cuya materialización se ha ligado a nuevos enfoques como la sostenibilidad, la integralidad y la socio-ecología, que van de la mano de políticas internacionales como los Objetivos del Desarrollo Sostenible y el más reciente Acuerdo de París sobre Cambio Climático (pactado en 2016).

Estos marcos políticos y estratégicos han introducido variables nuevas en los análisis ambientales del territorio, tales como servicios ambientales, tecnificación de la ruralidad y niveles de gobernanza, todas ellas válidas como recurso de conocimiento de los efec- tos de los proyectos de desarrollo sobre los territorios. Según lo plantea Romero en relación con el caso de la hidroelectricidad en Chile, dichas variables aún permanecen en un discurso que no es técnico sino político [19].

$\mathrm{Al}$ respecto, el interés de construir hidroeléctricas para posicionarse en el mercado energético ha hecho que el tiempo, así como el impulso económico de realizar la infraestructura en el menor tiempo posible, reduzcan estas variables a datos sesgados, grupos poblacionales selectivos y diagnósticos limitados en el tiempo. Con esto, la participación colectiva -a través de la cual se presentan las posibilidades de desarrollo local y regional- se invisibiliza, hecho que niega la posibilidad de establecer consensos alrededor de la infraestructura como aporte al desarrollo local; en cambio, esta sigue viéndose como único argumento de desarrollo.

Respecto a Hidroituango, comunidades organizadas en las Juntas de Acción Comunal de las veredas de los municipios afectados presentaron proyectos para el mejoramiento de vías e infraestructura a las empresas, en aras de participar en la inversión social del proyecto. Sin embargo, algunas de sus dificultades radicaron en la capacidad para formular propuestas y cumplir con los requerimientos de EPM, a la par con la vigencia de la normativa de los municipios. Esto derivó en un desarrollo incompleto, o la no participación directa de las comunidades en el territorio [1]. La investigación "Factores de desarrollo local en hábitats afectados por proyectos de desarrollo hidroeléctrico" [1] encontró, entre otros asuntos, que la infraestructura vial era uno de los elementos sobre los cuales se pedía mayor intervención al proyecto. Sin embargo, una vez este se ejecutó, no respondió a las necesidades locales de transporte y movilidad; al contrario, obedeció a dinámicas externas y lógicas económicas de mayor escala, o del proyecto mismo.

Comprender las afectaciones del territorio precisa un análisis de los comportamientos de los megaproyectos y su combinación con las experiencias de desarrollo local. Los denominados componentes del desarrollo -mejoramientos de infraestructura vial, equipamientos colectivos y construcción de vivienda para la reubicación de familias afectadas-, sumados a los estímulos económicos y apoyo técnico, se formalizan según las metodologías de la empresa ejecutora, de manera que las necesidades locales no suelen incorporarse, ni consiguen sumar elementos para el desarrollo local.

$\mathrm{El}$ acceso de las comunidades al conjunto de beneficios debe cumplir con requerimientos legales y organizativos que pueden estar por fuera de los esquemas de funcionamiento real del territorio. Ello deriva en inconsistencias, dada la dificultad que tienen las comunidades para la formulación de sus proyectos o la administración de los recursos asignados. La disparidad 
entre las aspiraciones de desarrollo de las comunidades y las propuestas de desarrollo referidas por los proyectos hidroeléctricos plantea una revisión de los procedimientos y metodologías con las cuales son abordadas las comunidades implicadas en los proyectos de desarrollo hidroeléctrico.

Se puede afirmar que el desarrollo de las comunidades campesinas e indígenas que habitan territorios con potenciales económicos no han participado directamente en la toma de decisiones de orden nacional o global. Con esto se afecta el derecho a la autonomía contemplada por la Ley 388 de 1997, la cual señala dentro de sus objetivos el establecimiento de mecanismos para:

1. Promover el ordenamiento del territorio.

2. Hacer uso equitativo y racional del suelo.

3. Preservar y defender el patrimonio ecológico y cultural localizado en el ámbito territorial.

D. Ejes de análisis ambiental en territorios afectador por proyectos hidroeléctricos

Tomando como punto de partida los objetivos planteados en la Ley 388 de 1997, se describen a continuación algunas inconsistencias de carácter metodológico identificadas en la planeación y ejecución del Proyecto Hidroeléctrico Ituango que podrían generalizarse a otros proyectos hidroeléctricos, con el fin de construir ejes de análisis que aporten a la lectura ambiental de los territorios.

$\mathrm{Al}$ respecto, se evidenció la manera como la toma de decisiones de las instituciones, sumada a la materialización de megainfraestructuras, transforman las condiciones ambientales y la vida de las comunidades, comprometiendo las lógicas de desarrollo local y participación de habitantes y entidades locales en la planificación futura del territorio. Esto quedó demostrado cuando el proyecto Hidroeléctrico Ituango declaró el estado de contingencia; según el informe de auditoría de cumplimiento realizado por la Contraloría General de la República,

Los habitantes afectados por la emergencia se encuentran [sic] en incertidumbre permanente frente a los riesgos que puede generar un evento de mayor magnitud al ocurrido, por un evento extremo de ruptura de la presa, sin poder regresar a sus hogares de manera tranquila y recuperar sus vidas. Sus derechos a estar completamente informados frente a la realidad del proyecto, están siendo vulnerados [20].

Se reconocen así tres determinantes por analizar en el territorio: escala, institucionalidad y construcción social y ambiental.

\section{Lectura de la escala}

Los diagnósticos previos realizados por las entidades ejecutoras no corresponden a las condiciones reales al materializarse un proyecto hidroeléctrico; estos se realizan con anticipación, o una vez iniciada la ejecución del proyecto. Las caracterizaciones se definen por las temporalidades del proyecto - planeación, ejecución y funcionamiento- también por la situación demográfica: condiciones socioeconómicas y de vulnerabilidad, número de habitantes, accesibilidad a servicios básicos. Indicadores que derivan de los procesos de participación, divulgación e información de las comunidades sobre los proyectos.

Las metodologías están orientadas a conocer el estado general del entorno. No obstante, los cambios en el territorio son heterogéneos, con poblaciones cambiantes y focos de desarrollo que, en principio, están ligados al proceso de construcción del proyecto -campamentos, carreteras, hospedajes y comercio- para satisfacer las necesidades de los trabajadores, las cuales varían según el avance que determine el mismo proceso constructivo del proyecto. En este orden, se observó que el territorio consta de múltiples escalas, las cuales van desde las dinámicas económicas y políticas extraterritoriales (gobierno y entidades multilaterales) hasta las relaciones cotidianas que desencadenan dinámicas productivas, organizativas y ecosistémicas.

La vida campesina, asociada a la agricultura, minería y producción artesanal, se vale de la infraestructura existente para acceder a bienes y servicios. No obstante, una vez estos se ven atravesados por la construcción de los proyectos hidroeléctricos, demandan otras construcciones y requerimientos técnicos que permitan hacer competitivos sus territorios frente a la economía que se instala alrededor de la generación de energía eléctrica. De no presentarse atención a las solicitudes del territorio en función de las relaciones agrícolas y de manufactura local, podría ocasionarse una pérdida de calidad de vida y un abandono paulatino de las actividades de aprovechamiento de las familias de su tierra.

Estas problemáticas están relacionadas con la baja capacidad que tienen los proyectos de involucrar a todos actores relacionados: al no considerarlos el eje central en su planificación y construcción, se llega a conflictos ambientales y sociales que no se han reflejado en los procesos de diagnóstico e implementación de planes y programas del proyecto hidroeléctrico en las diferentes áreas que le corresponden.

Como ejemplo de lo anterior, en Hidroituango se presentó una migración por trabajo: se crearon entre 2000 y 6000 empleos, y con ello aumentó la demanda de comercio y de servicios básicos. Esto exige permanentes cambios en el abordaje metodológico de los territorios, porque de no darse lo anterior se corre el riesgo de no atender todas las afectaciones que ocu- 
rren en el territorio, máxime cuando es previsible una emergencia ambiental y social de la escala mostrada en Hidroituango.

En razón de lo anterior, la socialización, los censos y la información secundaria de las comunidades deben obtenerse de un proceso efectivo de levantamiento de datos que garantice la disponibilidad de la mayoría de los habitantes, dada la movilidad de población y las nuevas economías que llegan con el proyecto (hoteles, restaurantes, mototaxis y servicios financieros y comerciales). A este nivel de intervención se requieren estudios que respondan a estas realidades complejas, cuyas variables den cuenta de los conflictos de los territorios a partir de lecturas rigurosas, hagan posible reflejar la cotidianidad trastocada por la inserción de los proyectos, y permitan una identificación del potencial social y productivo de los territorios.

La inconsistencia que se evidencia respecto a los diagnósticos y metodologías implementadas en el territorio está vinculada con la discrepancia entre los diagnósticos realizados y las realidades locales sobre las que se interviene. Esto se manifiesta en la selección de áreas de influencia que no consideran la totalidad del territorio a afectarse, o que sesgan las dinámicas que tienen como sistema territorial: ejemplos de ello se encuentran en la producción de café y el sistema de bosques y yacimientos de agua, al igual que el sistema de navegación y aprovechamiento del Río Cauca como un solo ecosistema.

La identificación de este problema de niveles de aproximación o conocimiento del territorio mediante las metodologías y su alcance sobre cuánto es afectado, permitió construir un eje de análisis basado en la escala: además de reconocer las áreas de influencia de los proyectos y los tratamientos que le correspondan, este hace posible escalar, de lo regional a lo local, las formas de habitar los lugares, las redes de comunicación, la asociatividad local y el intercambio que desde lo veredal o corregimental aportan, junto con su relevancia frente al desarrollo planteado por un megaproyecto.

\section{Coordinación interinstitucional}

Frente a la dificultad de participación de los habitantes al tomar decisiones, y en la definición de proyectos hidroeléctricos en sus territorios, se presenta la incapacidad de las instituciones y administraciones locales para vincular los proyectos a sus planes de desarrollo local e instrumentos de planificación.

En entrevistas realizadas a los secretarios de planeación en los municipios de Ituango y Toledo localizados en el área de influencia directa del proyecto hidroeléctrico, los funcionarios coinciden en que las dificultades principales para relacionarse con el proyecto radicaron en la desactualización de los planes básicos de ordenamiento territorial. Esto los llevó a definir sus programas y solicitudes bajo las orientaciones que las empresas ejecutoras de los proyectos hidroeléctricos implementan. Así, se tienen unos habitantes con limitaciones en cuanto a recursos para la planeación de sus municipios; instrumentos de gestión y de ordenamiento territorial; y acompañamiento de entidades de control y suministro de información de los territorios y proyectos.

Según el DNP, para el 2016 el 83\% de los municipios del país contaban con sus planes de ordenamiento territorial (POT) desactualizados; nótese al respecto que estos documentos se caracterizan por servir de soporte a todo proyecto, programa o transformación que se requiera implementar en el municipio. De ahí la necesidad de pensar los instrumentos en función de los acelerados cambios de los territorios, y en respuesta a las actividades económicas que se les imponen.

Las debilidades identificadas en materia de participación se alinean con la insuficiente incorporación de los proyectos de desarrollo de orden nacional en la planificación local. Esto por cuanto, al no estar en vigencia, los diagnósticos precisos en cuanto a usos del suelo, vocaciones productivas y gestión del riesgo pueden afectar el acceso a presupuesto y capacidad de gestión de recursos económicos generados por los proyectos hidroeléctricos a los municipios, así como la posibilidad de interlocución entre las entidades locales y proyectos de gran envergadura (tales como las hidroeléctricas o sistemas viales).

El proceso de abandono histórico por parte del Estado se ve reflejado en despojos, desplazamientos y actos violentos que han restringido el accionar de las administraciones locales, las juntas de acción comunal y cualquier organización comunitaria que pudiera tomar decisiones en relación con los proyectos de desarrollo hidroeléctrico o de otro tipo que se gestionen en el territorio. A pesar de esto, los proyectos de desarrollo se han dimensionado como intervenciones capaces de apaciguar el conflicto y una de las motivaciones de aceptación de las comunidades en sus territorios. Ello sitúa el acceso a desarrollo a cambio de una mitigación del conflicto y acceso al Estado, es decir, educación, salud y seguridad; no obstante, "(...) el proyecto hidroeléctrico es una infraestructura que al instalarse, limita con necesidades sociales e históricas que no han sido resueltas en tanto el conflicto que allí se manifiesta hace parte de la realidad geopolítica" [6].

El concepto de desarrollo basado en la provisión de infraestructura e intervención físico-espacial como medida de calidad de vida y superación del "atraso" de las poblaciones, ha implicado la modificación del paisaje al servicio del crecimiento económico, incluso por encima de los costos ambientales que puedan significar. Esto hace que la actual presión económica por incrementar las ganancias y utilidades del mercado de energía viabilice los proyectos hidroeléctricos cuya factibilidad se valora en función de los aportes económicos de su vida 
útil, más allá de las posibilidades locales de desarrollo y gestión de sus necesidades.

En tanto autoridad para el licenciamiento ambiental, la ANLA es conocedora de la viabilidad de los proyectos en función del cumplimiento de los términos de referencia definidos por la misma. Estas entidades permiten la continuidad de los proyectos y, como tales, son responsables directas de las metodologías de participación, presencia institucional y acompañamiento a las comunidades en el actuar de los proyectos hidroeléctricos.

En relación con el caso que nos ocupa, las comunidades han tenido la necesidad de expresarse en otros escenarios como consultas populares y audiencias públicas, y ante instancias como la Corte interamericana de Derechos Humanos y el Tribunal Latinoamericano del Agua. Esto obedece a que, en múltiples casos, las instituciones han desestimado la participación de campesinos y afectados por el proyecto, cuya información y capacidad es determinante para la coordinación entre instituciones y la elaboración de planes o estrategias que coadyuven a la salvaguarda de la biodiversidad y las comunidades vulnerables ante este tipo de iniciativas.

Los planes de manejo ambiental comportan tres propósitos complementarios: restitución integral de sus condiciones de vida con un entorno ambiental sustentado; restitución de redes sociales y culturales, y restitución y compensación de actividades económicas; su cumplimiento deja un interrogante frente a la efectividad de las instituciones en el territorio, y al modo en que sus estrategias han garantizado un proceso coherente entre entidades de diferentes escalas para satisfacer tales metas. De ahí que el eje de coordinación interinstitucional sirva como fundamento para atender las afectaciones y necesidades de los habitantes.

3. La construcción social y ambiental del territorio Las intervenciones civiles que se desligan de las realidades históricas sociales y geográficas del territorio afectan el proceso de participación en el desarrollo del proyecto hidroeléctrico, así como su continuidad con las comunidades en el territorio mismo.

La planeación y ejecución de los proyectos de desarrollo del sector energético ha convertido la participación de las comunidades locales en un hecho secundario, pues sus decisiones han sido relegadas a una descripción de los sujetos en los entornos por fuera de sus dinámicas históricas; es decir, sin un reconocimiento de la memoria y significados de los territorios. Estas acciones han transformado el objeto técnico para el desarrollo -represas, vías, puentes- en protagonistas encargados de trazar la historia de los territorios, con lo cual anulan incluso las realidades preexistentes en los mismos (cuya permanencia en el territorio se refleja, por ejemplo, en la pesca, la siembra y la minería artesanal).
La permanente combinación de actividades económicas y sociales se vincula, a su vez, a las dinámicas de los ríos y los ritmos de los ecosistemas, los cuales deberían ser entendidos y modificados mientras se dé una participación real y directa por parte de quienes han habitado y convivido en estos territorios. Así, el desarrollo, entendido como proceso de incorporación de los potenciales y capacidades locales, además de aportar al mejoramiento de las condiciones de vida, debe involucrarse con los procesos históricos y económicos propios del lugar donde se sitúa. Esto también compete al reconocimiento de los conflictos, violencias y estigmatizaciones que los territorios puedan padecer.

Visto lo anterior, el Plan Integral de la Hidroeléctrica Ituango integraba las relaciones ambientales y sociales sin resolver las desigualdades en términos de los mínimos organizativos de las comunidades y administraciones locales. Con ello se limitaron el desarrollo y las condiciones necesarias para que el proyecto pudiera articularse, implicarse y hacer parte del proceso del que forman parte quienes han ocupado históricamente el territorio intervenido. Parte de estas dificultades radica en la idea de participación como mecanismo de información, como se muestra en el "Resumen de revisión ambiental y social" presentado por el BID sobre Hidroituango en el año 2016: allí se ubica la participación social como un proceso "(...) cuyo objetivo principal es el de mantenerlas [a las comunidades] informadas sobre los avances del Proyecto y manejar sus expectativas" [21]. Esto deja constancia del lugar que tienen estas personas como actores pasivos en el proceso de desarrollo del proyecto.

Un ejemplo más claro de este hecho tiene que ver con la comunicación por parte del proyecto sobre la no existencia de etnias, ni comunidades indígenas o afrodescendientes, en el área de influencia del proyecto hidroeléctrico Ituango [11]. Esto implicó la no realización de consultas previas e inclusión de minorías, a pesar de que en la región habitan los indígenas Emberá Katios del resguardo Jaidukama, quienes también se sirven del Río Cauca y, por tanto, podrían considerarse población afectada por la construcción de la represa. Tal decisión retiró a dicha comunidad del proceso de planificación y acceso a recursos concebido en el marco del proyecto, que de igual manera se instaló en su territorio.

$\mathrm{Al}$ respecto, se plantea aquí el proceso de construcción ambiental y social del territorio como eje articulador entre la planificación del territorio y las institucionalidades que intervienen en él. Aunque se tienen mecanismos de participación y políticas de inclusión en los territorios, la lógica de programas y lineamientos no ha alcanzado el propósito de participación en la amplitud que lo exige el artículo 79 de la Constitución Política de Colombia: 
Todas las personas tienen derecho a gozar de un ambiente sano. La ley garantizará la participación de la comunidad en las decisiones que puedan afectarlo [22].

Se considera que dichas garantías yacen en la formulación, planificación y ejecución de megaproyectos que incorporen las necesidades locales y los procesos de construcción social que se han dado en el territorio como base fundamental para orientar ejercicios de intervención, como los que se presentan en las hidroeléctricas.

\section{Conclusiones}

Respecto a Hidroituango se puede concluir que con el monitoreo del movimiento de los macizos rocosos y la revisión constante del comportamiento del Río Cauca, sumado a los trabajos de conclusión de la presa propios del manejo técnico del proyecto, se precisa conocer la planificación que se busca con las comunidades; las alternativas y recuperación de las actividades económicas que se tienen previstas; los procesos de desarrollo que contemplan la gestión del riesgo en los ámbitos local e individual; y la forma como se promoverá su conocimiento para que los habitantes del territorio convivan con él durante el resto de sus vidas (o bien los 50 años en que el proyecto permanezca en el territorio).

Lo anterior implica verificar los planes de atención a la fauna y flora afectada; mitigar los impactos en áreas no identificadas como de influencia del proyecto, hoy alteradas (como La Mojana en los departamentos de Córdoba y Sucre); y comunicar y garantizar la participación de todos los actores sobre el futuro del proyecto. En este punto es preciso tomar consciencia de que ya se hizo una modificación física del Río Cauca, que debe ser monitoreada en aras de mitigar daños y efectos no deseados; por tanto, y en tanto integración de la población, el ecosistema ambiental debe tender a un nuevo equilibrio, que reivindique el tejido social alterado por los megaproyectos.

Plantear una crítica al modelo de desarrollo que orienta los proyectos hidroeléctricos no significa negarlo, máxime si corresponde pensar en categorías y ejes de análisis diferentes a los establecidos por las entidades que promueven los proyectos hidroeléctricos al servicio de las dinámicas económicas globales, por medio de las cuales se priorice y canalice la capacidad de movilización de recursos humanos y físicos para la materialización del desarrollo en función de una sinergia entre las relaciones locales-globales, que hoy se le imponen a los territorios.

La globalización hoy debe aceptarse por cuanto su manifestación en el territorio es real y lo ha modificado en todas sus escalas: ejemplo de ello es la producción de energía para la exportación a partir de hidroeléctricas, por la cual regiones -incluso aisladas- dentro de un país consiguen conectarse mediante redes eléctricas a otras ciudades del país y el continente. Su existencia como proceso de desarrollo es innegable; sin embargo, no es el único indicativo de desarrollo social que existe.

En el eje de análisis asociado a la escala se tiene una base para reconocer el alcance de una intervención física en el ambiente. La concepción de homogenizar el territorio bajo una única actividad productiva, como la generación de hidroelectricidad, restringe las alternativas de desarrollo del territorio y da lugar a incertidumbres para quienes lo habitan. Por tanto, más allá de ser símbolo de desarrollo, una mirada a las hidroeléctricas posibilita la integralidad de la ingeniería y la lectura ambiental de la misma; valorar su planificación y ejecución como un hecho político, que define y orienta la vida de comunidades y territorios, abre la frontera del desarrollo a los procesos sociales y su conjunción con la naturaleza.

Los megaproyectos resultan por decisiones políticas: sus marcos normativos pueden ajustarse a los cambios que la sociedad exige y, con ello, abrir posibilidades de orientación del desarrollo en el territorio, en respuesta a las capacidades locales, de sus comunidades, sus instituciones y organizaciones. Los proyectos hidroeléctricos en Colombia implican hoy una reestructuración de los instrumentos públicos, dada la transgresión de las relaciones locales y de participación que han evidenciado. En este sentido, corresponde reivindicar por un lado mecanismos como la consulta previa de tal manera que, además de ser un espacio para la toma de decisiones de las comunidades étnicas, se dé participación en ellas a comunidades campesinas que han habitado históricamente los territorios; y por otro, la consulta popular, de suerte tal que con ellas sea posible definir el futuro de los municipios y la protección de actividades que afectan la autonomía territorial, todo ello junto con una revaluación colateral del derecho del ente nacional de actuar por encima de los intereses de las entidades locales y regionales.

El tercer eje de análisis, de construcción social y ambiental del territorio, da un reconocimiento al lugar que ha sido socialmente construido, con particularidades históricas y significados que se han manifestado y combinado con los conflictos sociales, que como tales tienen efectos en la economía local. Aun cuando los proyectos de vida alrededor de estas prácticas respondan a entornos de naturaleza rural o ajena a la ciudad, exigen respeto y reconocimiento como proceso de vida y desarrollo. En consecuencia, se propone desde aquí exigir a los promotores de megaproyectos la elaboración contextualizada de instrumentos de planificación y metodologías para la intervención de los territorios, que reconozcan a las comunidades y la existencia de otras posibilidades de desarrollo en lo local, así como de intervención ambiental con el menor impacto en los ecosistemas, con lo cual se dé margen a la recuperación 
de los entornos naturales para la vida salvaje en equilibrio con las fuerzas que los degradan. Así entonces, los territorios pueden potenciar sus capacidades por fuera de los megaproyectos; en este sentido, no todos los espacios habitados requieren altas concentraciones de infraestructura para propiciar su propio bienestar, incluso como medida de salvaguarda de la naturaleza -hoy amenazada por múltiples acciones para la circulación de capital-.

El debate alrededor del desarrollo es una tarea conjunta de toda la sociedad, la cual requiere importantes consensos sobre el futuro del territorio. En esta línea se recomienda abrir espacios de discusión que impliquen a todos los saberes locales, y en los que converjan naciones indígenas, comunidades campesinas e instituciones de la academia y el Estado, a fin de generar cambios en los marcos normativos y legislativos, así como en la concepción de desarrollo que se profesa desde las aulas. Esta es una tarea de largo plazo, que debe comenzar ad portas de una crisis ambiental derivada de nuestras decisiones políticas.

\section{Trabajos futuros}

Se recomienda profundizar en estudios alrededor de la eficiencia energética que permitan transitar a otros medios de producción de energía, en procura de que las comunidades receptoras sean partícipes de este cambio. La construcción de políticas y marcos normativos y metodológicos alrededor de la construcción de los megaproyectos es un tema aún condicionado por las orientaciones económicas; como tal, debe abordarse desde un enfoque científico y conceptual que contribuya a la generación de nuevas propuestas de marcos normativos por medio de los cuales se legisle sobre los hechos ambientales en el territorio.

\section{Referencias}

[1] I.N. Cuervo López, "Factores de desarrollo local en un hábitat afectado por proyectos de desarrollo hidroeléctrico: proyecto hidroeléctrico ituango, antioquia, colombia." Universidad Nacional de Colombia, Sede Medellín, 2018.

[2] C. A. Medina, A. B. Hernández, and M. A. Fuentes, "Evaluación de sostenibilidad en proyectos de desarrollo," Ingenierías USBMed, vol. 10, no. 1, pp. 3439, 2019.

[3] World Energy Council, "Hydropower." Energy resources, 2016. [Online]. Disponible: https://www.worldenergy.org/data/resources/ resource/hydropower/.[Último acceso: 20-Sep2003].

[4] F. Ortúzar, "Desmantelando el mito de las grandes represas tomado." 2014, [Online]. Disponible: http://www.aida-americas.org/es/blog/ desmantelando-el-mito-de-las-grandes-represas.

[5] Ministerio de Ambiente Vivienda y Desarrollo Territorial, "Metodología general para la presentación de estudios ambientales." Bogotá, 2010.

[6] J. A. Polanco, "La responsabilidad social del grupo epm: una nueva postura política frente al territorio," Cuadernos de administración, vol. 27, no. 49, pp. 65-85, 2014.

[7] ANLA, "Terminos de referencia para la elaboración del estudio de impacto ambiental-eia en proyectos de construcción y operación de centrales generadores de energía hidroeléctrica." Bogotá D.C., 2017. Disponible: http://portal.anla.gov. $\mathrm{co} /$ sites/default/files/comunicaciones/SIPTA/ Terminos_referencia/terminos_de_referencia_ eia_hidroelectricas.pdf.

[8] Hidroelectrica Ituango, "Manual de valores unitarios. avaluó de predios y mejoras." Bogotá D.C., 2010. Disponible: https://www.hidroituango.com.co/documentos/ Administrativos/Manuales_politica/Manual_ de_valores_Hidroituango_parte_1.pdf.

[9] G. D. Valencia Agudelo and C. A. Vasco Correa, "La interconexión eléctrica de las américas," Perf. Coyunt. Económica, vol. 1, no. 19, pp. 93-11, 2012.

[10] Hidroelectrica Ituango, "Proyecto hidroeléctrico ituango una contribución al desarrollo local y regional un mejor futuro para los colombianos." 2016. Disponible: http://www.hidroituango.com.co/ proyectos/proyecto-hidroelectrico-ituango/38. [Último acceso: 01-Oct-2017].

[11] E. Villamizar, "Hidroeléctrica ituango." Resultados del análisis causa raíz, 2018. Disponible: https://www.hidroituango.com.co/articulo/ cumpliendo-con-el-compromiso-de-informar-a-laopinion-publica-y-en-un-ejercicio-de-transparencia -epm-compartio-los-resultados-del-analisis-causaraiz-fisica-que-dio-origen-a-la-contingencia-en-elproyecto-hidroelectrico-ituango/495. [Último acceso: 05-Mar-2018].

[12] Semana Sostenible, "Histórico: declaran al río cauca como sujeto de derechos." Revista Semana, 20-Jun-2019. https://sostenibilidad. semana.com/medio-ambiente/articulo/ historico-declaran-al-rio-cauca-como-sujeto-de -derechos/44662.

[13] P. McCully, Ríos silenciados: ecología y política de las grandes represas. Buenos Aires: ProtegerAmigos de la Tierra, 2004.

[14] B. Chellaney, Water, peace, and war: Confronting the global water crisis. Londres y Nueva York: Rowman \& Littlefield, 2013.

[15] C. A. S. Montero and A. M. C. Forero, "Expansión 
hidroeléctrica, estado y economías campesinas: el caso de la represa del quimbo, huila-colombia," Mundo agrario, vol. 16, no. 31, 2015.

[16] T. Roa and B. Duarte, "Desarrollo hidroeletrico, despojo y transformacion territorial: el caso de hidrosoamoso, santander, colombia." in Aguas Robdas. Despojo Hídrico y Movilización Social., A. Arroyo and R. Boelens, Eds. Quito: Abya Yala, 2013, pp. 1-43. https://totumasymaracas.files.wordpress.com/ 2013/10/doc_tati-bibi_art-hidrosogamoso_ aguas-robadas_2013_rfinal.pdf.

[17] S. E. Hoyos-Gómez and R. Bernal, "Rheophytes of the samaná norte river, colombia: a hydroelectric project threatens an endemic flora," Tropical Conservation Science, vol. 11, pp. 11-13, 2018.

[18] . Congreso de la República de Colombia. Decreto 2811 de 1974. Colombia.

[19] H. Romero Toledo, "Ecología política y represas: elementos para el análisis del proyecto hidroaysén en la patagonia chilena," Revista de Geografía Norte Grande, vol. 175, pp. 161-175, 2014.

[20] Contraloría General de la República, "Informe auditoría de cumplimiento gestión de las autoridades ambientales en el proceso de licenciamiento-proyecto hidroeléctrico ituango." Bogotá, 2018. https: //issuu.com/miltonalfonsogranadossguerra/ docs/contralor_a_hidro-ituango.

[21] Corporación interaméricana de Inversiones (CII), "Resumen de revisión social y ambiental (esrs, por sus siglas en inglés), proyecto hidroeléctrico ituango." 2016. file://C:/Users/yohana.lopez/ Downloads/esrs_ituango_1\%20(2).pdf.

[22] Presidencia de la República de Colombia, "Constitución política de colombia." 2014. [En línea]. Disponible: http: //wsp.presidencia.gov.co/Normativa/Documents/ Constitucion-Politica-Colombia.pdf. [Último acceso: Julio 2019]. 